\title{
Natural Convection in an Inclined Square Cavity with Heated and Cooled Adjacent Walls and Filled with a Porous Medium
}

\author{
C. Revnic ${ }^{1}$, I. Pop ${ }^{*}, 2$ T. Grosan ${ }^{2}$ and D.B. Ingham ${ }^{3}$ \\ ${ }^{I}$ Faculty of Pharmacy, University of Medicine and Pharmacy, Cluj-Napoca, Romania \\ ${ }^{2}$ Babeş-Bolyai University, Department of Applied Mathematics, R-3400 Cluj-Napoca, CP 253, Romania \\ ${ }^{3}$ Centre for Computational Fluid Dynamics, University of Leeds, Leeds LS2 9JT, UK
}

\begin{abstract}
Steady natural convection flow in an inclined square cavity filled with a porous medium has been investigated. It is assumed that the cavity is heated on one wall and cooled from an adjacent wall, while the other two walls are adiabatic. The governing dimensionless partial differential equations have been solved numerically for the stream function, isotherms, and the local and average Nusselt numbers using a central finite-difference method and Richardson extrapolation. Effects of the various parameters on the flow and heat transfer characteristics have been investigated, namely: Rayleigh numbers 10,100 , and 1000 , and the inclined angle of the cavity from $0^{\circ}$ to $315^{\circ}$. Special attention is given to the understanding of the effects of the inclination on the heat transfer rates on the heated and cooled walls as well as the flow configurations. It is found that the streamlines, isotherms and the average Nusselt numbers are affected significantly by the inclination of the cavity and the Rayleigh number. Results could be obtained for angles of inclination for Rayleigh numbers up to 100 but for a Rayleigh number of 1000 solutions could not be obtained for all angles of inclination.
\end{abstract}

Keywords: Natural convection, inclined square cavity, porous medium, numerical results.

\section{INTRODUCTION}

Over the last few years, the analysis of natural convection in enclosures filled with fluid-saturated porous media has been studied extensively using different geometries, equation models, and numerical techniques and can be used in numerous applications of engineering. Some of these applications are solar power collectors, geothermal applications and nuclear reactors. Detailed reviews on the subject of porous media can be found in the welldocumented research books by Nakayama [1], Nield and Bejan [2], Ingham and Pop [3], Vafai [4], Lemos [5], Vadasz [6], etc. In the past few years, most of the researchers have focused on the investigation of natural convection in a porous square or rectangular enclosure with constant temperature or heat flux boundary conditions, see for example Bejan [7], Goyeau et al. [8], Gross et al. [9], Manole and Lage [10], Saeid and Pop [11], Baytas and Pop [12], etc. However, different boundary conditions are also important in practice due to the fact that a good knowledge of the flow field and temperature distributions assist in the design of high efficient thermal systems. The direction of the temperature gradient with respect to the gravity associated with the heating of the walls is also a primary factor in the formation of the velocity and temperature fields.

The research regarding natural convection in cavities with differentially heated neighbouring walls is limited.

*Address correspondence to this author at the Babeş-Bolyai University, Department of Applied Mathematics, R-3400 Cluj-Napoca, CP 253, Romania; Tel: +40 264594 315; Fax: +40 264591 906;

E-mail:pop.ioan@yahoo.co.uk
Anderson and Lauriat [13] have studied, both experimentally and numerically, the natural convection in a square cavity heated from below and cooled from adjacent walls. Kimura and Bejan [14] numerically investigated the natural convection inside a right-angled corner formed by a hot vertical wall and a cold horizontal wall. Ganzarolli and Milanez [15] studied numerically the natural convection in a rectangular enclosure heated from below and symmetrically cooled from both side walls. The heat transfer characteristics of the natural convection in square and rectangular enclosures heated and cooled on adjacent walls has also been studied by Aydin et al. [16, 17] and Rahman and Sharif [18]. Finally, we mention the paper by Ece and Büyük [19] on the natural convection flow of a viscous (Newtonian) fluid in the presence of a magnetic field in an inclined rectangular enclosure heated from one side wall and cooled from an adjacent wall. The basic dimensionless equations are solved numerically using the finite-difference method along with the Richardson extrapolation technique. Streamlines, isotherms, and local Nusselt number have been determined and discussed.

\section{PHYSICAL MODEL AND GOVERNING EQUATIONS}

A schematic of the two-dimensional inclined square cavity filled with a fluid-saturated porous medium is shown in Fig. (1), where $\bar{x}$ and $\bar{y}$ are the Cartesian coordinates, $L$ is the length of the cavity and $\phi$ is the inclination angle from the horizontal. It is assumed that the right hand side and bottom walls of the cavity are adiabatic, and the fluidsaturated porous medium is isothermally heated and cooled by the left hand side and top walls at uniform temperatures $T_{h}$ and $T_{c}$, respectively, where $T_{h}>T_{c}$. All walls are 
impermeable and the flow is assumed to be steady. Constant fluid-porous medium properties are assumed, except for the density changes with temperature that induce buoyancy forces, i.e. the Boussinesq approximation is adopted. Under these assumptions, the continuity, Darcy and energy equations can be written in dimensional form as follows:

$\frac{\partial \bar{u}}{\partial \bar{x}}+\frac{\partial \bar{v}}{\partial \bar{y}}=0$

$\frac{\partial \bar{u}}{\partial \bar{y}}-\frac{\partial \bar{v}}{\partial \bar{x}}=\frac{g K \beta}{v}\left(\frac{\partial T}{\partial \bar{y}} \sin \varphi-\frac{\partial T}{\partial \bar{x}} \cos \varphi\right)$

$\bar{u} \frac{\partial T}{\partial \bar{x}}+\bar{v} \frac{\partial T}{\partial \bar{y}}=\alpha_{m}\left(\frac{\partial^{2} T}{\partial \bar{x}^{-2}}+\frac{\partial^{2} T}{\partial \bar{y}^{2}}\right)$

where $\bar{u}$ and $\bar{v}$ are the velocity components along the $\bar{x}$ and $\bar{y}$ directions, respectively, $T$ is the fluid temperature, $g$ is the magnitude of the acceleration due to gravity, $K$ is the permeability of the porous medium, $\alpha_{m}$ is the effective thermal diffusivity of the porous medium, $\beta$ is the thermal expansion coefficient, and $v$ is the kinematics viscosity.

We introduce the following dimensionless variables:

$$
\begin{gathered}
x=\bar{x} / L, y=\bar{y} / L, u=\left(L / \alpha_{m}\right) \bar{u}, v=\left(L / \alpha_{m}\right) \bar{v} \\
\theta=\left(T-T_{0}\right) /\left(T_{h}-T_{C}\right)
\end{gathered}
$$

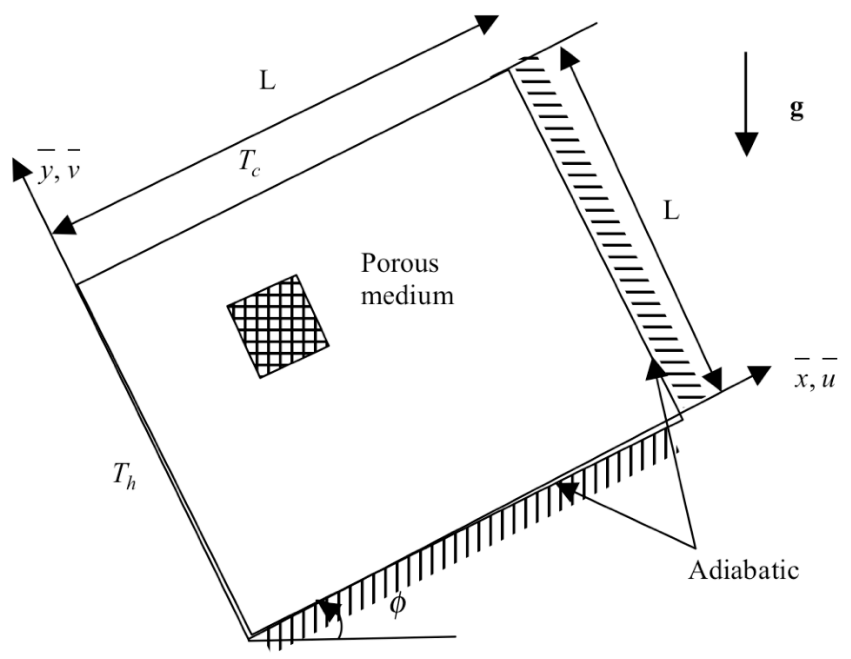

Fig. (1). Physical model and coordinate system.

where $T_{0}$ is a characteristic temperature and we assume that $T_{0}=\left(T_{h}+T_{c}\right) / 2$. Also we introduce the dimensionless stream function $\psi$ defined in the usual way as $u=\partial \psi / \partial y$ and $v=-\partial \psi / \partial x$. Thus, Eqs. (1) - (3) can be written in dimensionless form as follows:

$$
\frac{\partial^{2} \psi}{\partial y^{2}}+\frac{\partial^{2} \psi}{\partial x^{2}}=R a\left(\frac{\partial \theta}{\partial y} \sin \phi-\frac{\partial \theta}{\partial x} \cos \phi\right)
$$

$\frac{\partial \psi}{\partial y} \frac{\partial \theta}{\partial x}-\frac{\partial \psi}{\partial x} \frac{\partial \theta}{\partial y}=\frac{\partial^{2} \theta}{\partial x^{2}}+\frac{\partial^{2} \theta}{\partial y^{2}}$

where $R a=g K \beta\left(T_{h}-T_{c}\right) L / v \alpha_{m}$ is the Rayleigh number for the porous medium. According to the boundary conditions defined in Fig. (1), Eqs. (5) and (6) have to be solved subject to the following boundary conditions:

$\psi(x, 0)=0, \psi(0, y)=0, \psi(x, 1)=0, \psi(1, y)=0$

$\theta(0, y)=\frac{1}{2}, \theta(x, 1)=-\frac{1}{2}, \frac{\partial \theta}{\partial y}(x, 0)=0, \frac{\partial \theta}{\partial x}(1, y)=0$

The physical quantities of interest are the local and average Nusselt numbers from the heated wall, $N u_{h}(y)$ and $\overline{N u}_{h}$, and cooled wall, $N u_{c}(y)$ and $\overline{N u}_{c}$, respectively, which are given by

$$
\begin{aligned}
& N u_{h}(y)=-2 \frac{\partial \theta}{\partial x}(0, y), \overline{N u} h=\int_{0}^{1} N u_{h}(y) d y \\
& N u_{c}(x)=-2 \frac{\partial \theta}{\partial y}(x, 1), \overline{N u}_{c}=\int_{0}^{1} N u_{c}(x) d x
\end{aligned}
$$

\section{RESULTS AND DISCUSSION}

In order to obtain the numerical solution of Eqs (5) and (6), a central finite-difference scheme has been used and the system of discretised equations have been solved using a Gauss-Seidel iteration technique. The unknown quantities $\psi$ and $\theta$ are calculated iteratively until the following criterion of convergence is fulfilled:

$\sum_{i, j}\left|\chi_{\text {new }}(i, j)-\chi_{\text {old }}(i, j)\right| \leq \varepsilon$

where $\chi$ represents the temperature or the stream function and $\varepsilon$ is the convergence criterion. In all the results presented in this paper, we have found that $\varepsilon=10^{-7}$ is sufficiently small such that any smaller value produces results which were graphically indistinguishable. In order to choose the size of the grid, accuracy tests using the central finite-difference method and Richardson extrapolation [20] for mesh sensitivity analysis has been performed for $R a=100$ for some typical values of the stream function and temperature using three sets of grids: $26 \times 26,51 \times 51$ and $101 \times 101$ as shown in Table 1 . Reasonably good agreement has been found between the results obtained using the $51 \times 51$ and $101 \times 101$ grids and the extrapolated results. Therefore, all the results presented in this paper are those obtained using the extrapolated results. Due to the lack of suitable results in the literature, we are unfortunate in not being able to compare the present results with other results. However, because the numerical method has been used in our recently published papers [21] and [22], we are confident that the present results are accurate. Further, our results are comparable with the ones reported by Aydin et al. [16] for a similar geometrical cavity filled with viscous and incompressible fluid (non-porous medium). It should be noted that the value of $R a=100$ is a typical value that has been used by other authors, see for example [23], for a cavity filled with a fluid-saturated porous medium. Thus, we have 
concentrated our investigations on Rayleigh numbers in this vicinity.

Table 1. Some Typical Values of the Stream Functions and Temperature for $R a=100, \varphi=0^{\circ}$

\begin{tabular}{|c|c|c|}
\hline Nodes & $\boldsymbol{\psi ( \mathbf { 0 . 2 4 } ; \mathbf { 0 . 2 4 } )}$ & $\boldsymbol{\theta}(\mathbf{0 . 2 4 , 0 . 2 4 )}$ \\
\hline \hline $26 \times 26$ & -3.8460 & -0.1478 \\
\hline $51 \times 51$ & -3.8795 & -0.1485 \\
\hline $101 \times 101$ & -3.8884 & -0.1497 \\
\hline Richardson extrapolation & -3.8913 & -0.1501 \\
\hline
\end{tabular}

Streamlines and isotherms are shown in Figs. (2, 3) for $R a=10$ (Fig. 2) and for $R a=100$ (Fig. 3) and for values of the inclined angle of $\phi=0,45^{\circ}, 90^{\circ}, 135^{\circ}, 180^{\circ}, 225^{\circ}$, $270^{\circ}, 315^{\circ}$. Streamlines are plotted on the left hand side, while isotherms are plotted on the right hand side of these figures. It is clear from Fig. (1) that certain values of the inclined angles represent different physical problems and this leads to variations in the patterns of the streamlines and isotherms. While a cavity with $\phi=0^{\circ}$ represents heated from one side and cooled from above, a cavity with $\phi=90^{\circ}$ corresponds to the problem of heated from below and cooled from one side. The other two cases include heated from one side and cooled from below when $\phi=180^{\circ}$, and heated from above and cooled from one side when $\phi=270^{\circ}$. It is observed that a unicellular recirculation flow pattern appears for all the inclined angles, except for $\phi=45^{\circ}$ and $225^{\circ}$ where two bicellular recirculation flow patterns are observed. However, we observe, as expected, that there is a symmetrical pattern for the cases $\phi=45^{\circ}$ and $225^{\circ}$. On the other hand, the solutions for $\phi=180^{\circ}$ and $\phi=270^{\circ}$ are identical, after a suitable rotation about the centre of the cavity and, as expected, the flow for $\phi=45^{\circ}$ and $R a=100$ is symmetrical. Further, it is observed that the nature of the streamlines and isotherms do not change significantly as the angle of inclination changes. The buoyancy force which ascends the fluid particle heated near the hot wall acts parallel to this wall when the cavity is inclined at $\phi=0^{\circ}, 90^{\circ}, 180^{\circ}$ and $270^{\circ}$ but forces them towards the hot and cold walls when the cavity is inclined at $\varphi=45^{\circ}, 135^{\circ}, 225^{\circ}$, and $315^{\circ}$ and away from the hot and cold walls for all the other angles of inclination. As regards the isotherms, it is observed that they are almost the same as for $R a=10$ as $R a$ increases, while they are closer to each other near the hot or cold walls depending on the angle of inclination. This indicates that there is a higher heat flux from the hot wall to the cold wall and vice versa. This higher heat flux depends on the angle of inclination and it is more pronounced for higher angles of inclination. Further, it can be seen that as $R a$ increases then the isotherms are bulged near the hot wall and squeezed near the cold wall, and vice versa depending on the angle of inclination. The streamlines are displayed in two symmetric counter-rotating cells when the cavity is tilted at the angles $\phi=45^{\circ}$ and $225^{\circ}$ for $R a=10$ and $R a=100$. The upper one rotates counterclockwise, while the lower one rotates clockwise, see Figs. (2b, f, 3b, f). It is observed that for $R a=1000$ we are able to determine the flow and heat transfer only for $\phi=180^{\circ}$. It appears that for other values of $\phi$, the flow becomes unsteady due to the destabilizing effects of the isothermal walls. In fact, the flow field becomes threedimensional beyond $R a=1000$ at all angles of inclination. Further, it should be observed that a boundary layer is formed near the hot wall for $R a=100$ and the angle of inclination $\phi=315^{\circ}$ and it is more pronounced for $R a=1000$ when $\phi=180^{\circ}$, see Figs. $(\mathbf{3 h}, \mathbf{4})$.

The variations of the local Nusselt number $N u_{h}$ for the hot wall, and $N u_{c}$ for the cold wall, with the angle of inclination are illustrated in Figs. $(\mathbf{5}, \mathbf{6})$. It is observed that $N u_{h}$ continuously increases along the hot wall for all the values of $\phi$ considered, except $\phi=0^{\circ}$ and $315^{\circ}$, see Figs. (5a, 6a). However, $N u_{c}$ first decreases and then increases along the cold wall for $\phi=45^{\circ}, 90^{\circ}$ and $135^{\circ}$ and for all the other values of $\phi$ it continuously decreases, see Figs. (5b, 6b). This is in accordance with the distribution of the isotherms shown in Figs. (2, 3).

Finally, the obtained numerical results for the average Nusselt number $\bar{N} u_{h}$ and $\bar{N} u_{c}$ for the hot and cold walls using both the second-order finite-difference method and Richardson extrapolation are given in Tables $\mathbf{2}$ and $\mathbf{3}$ when $R a=100$ and for different values of the angles of inclination. It is observed that, as expected, the values of the average Nusselt numbers, $\bar{N} u_{h}$ and $\bar{N} u_{c}$ are almost equal when $\phi=225^{\circ}$ (the largest difference between the values of the Nusselt numbers on the cooled and heated walls is less than 1.5\%). Further, it can be observed that both $\bar{N} u_{h}$ and $\bar{N} u_{c}$ increase and decrease simultaneously. Starting from a maximum value at $\phi=0^{\circ}$ the Nusselt number $\overline{N u}_{h}$ gradually decreases with $\phi$ and then increases for $\phi=90^{\circ}$. Further, they smoothly decrease to a minimum at $\phi=225^{\circ}$. The maximum values of both $\bar{N} u_{h}$ and $\bar{N} u_{c}$ are attained at $\phi=0^{\circ}$ and $\phi=90^{\circ}$, respectively, and the energy transport increases due to the convective effect of the isothermal cold wall. It is observed that the values of the average Nusselt numbers, $\bar{N} u_{h}$ and $\bar{N} u_{c}$, are almost equal. 
(a)
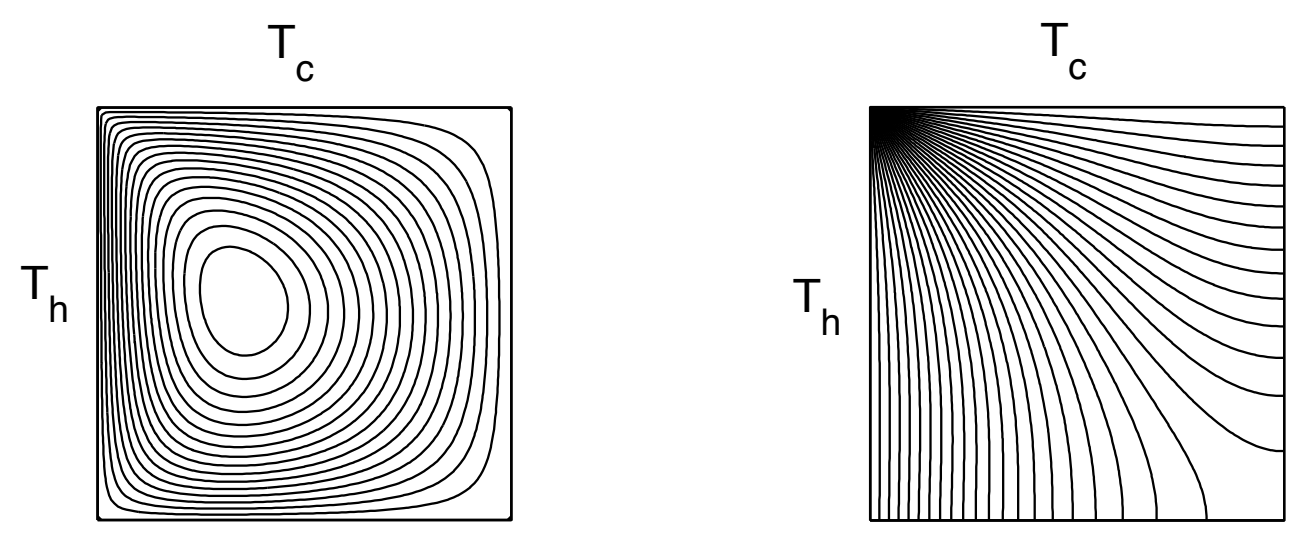

(b)
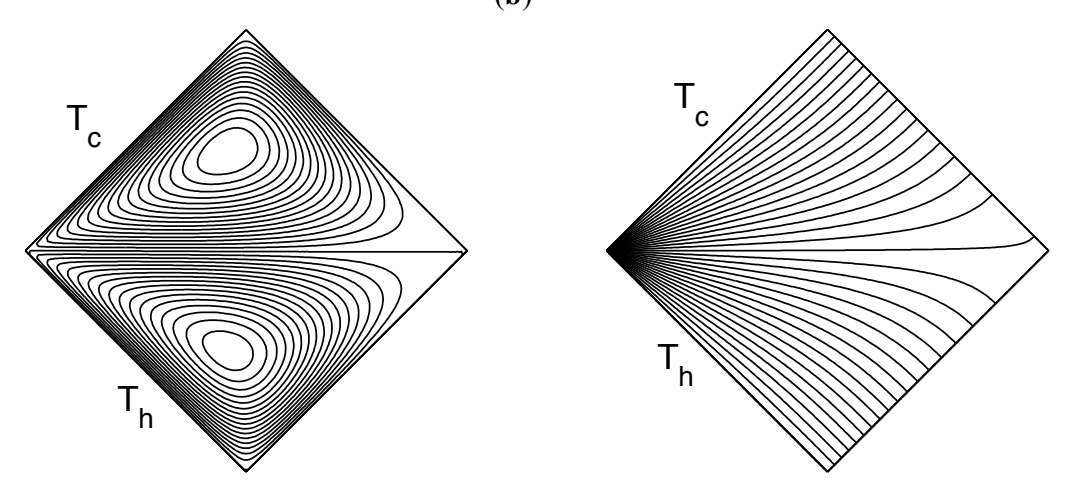

(c)
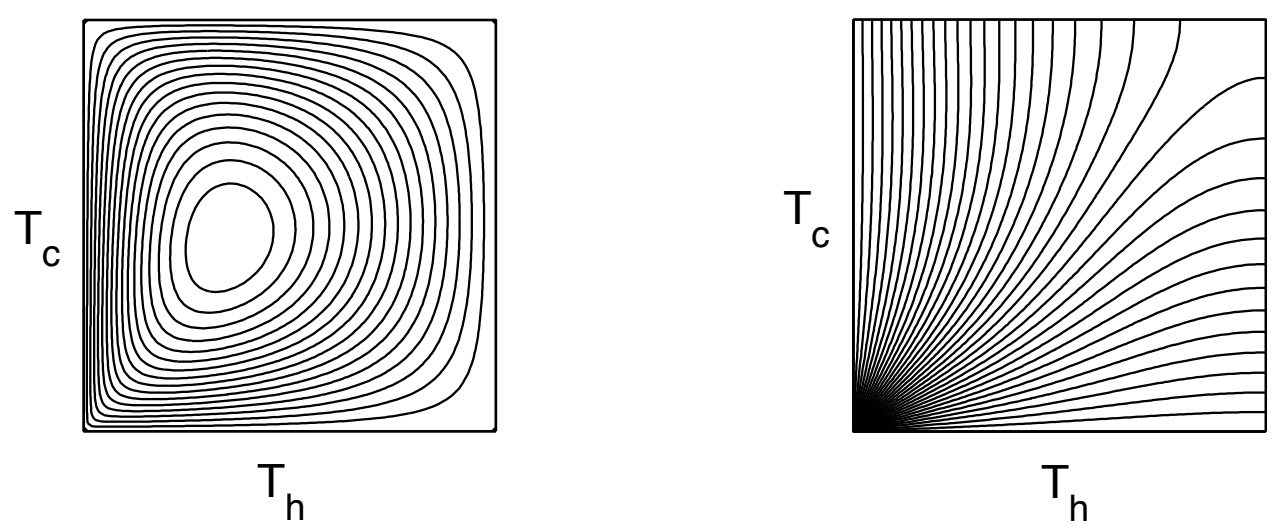

(d)
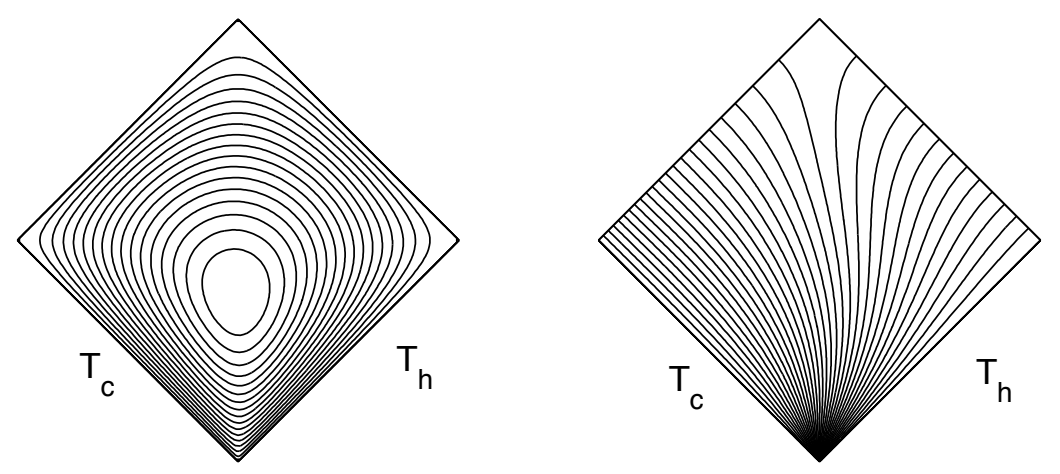
(e)
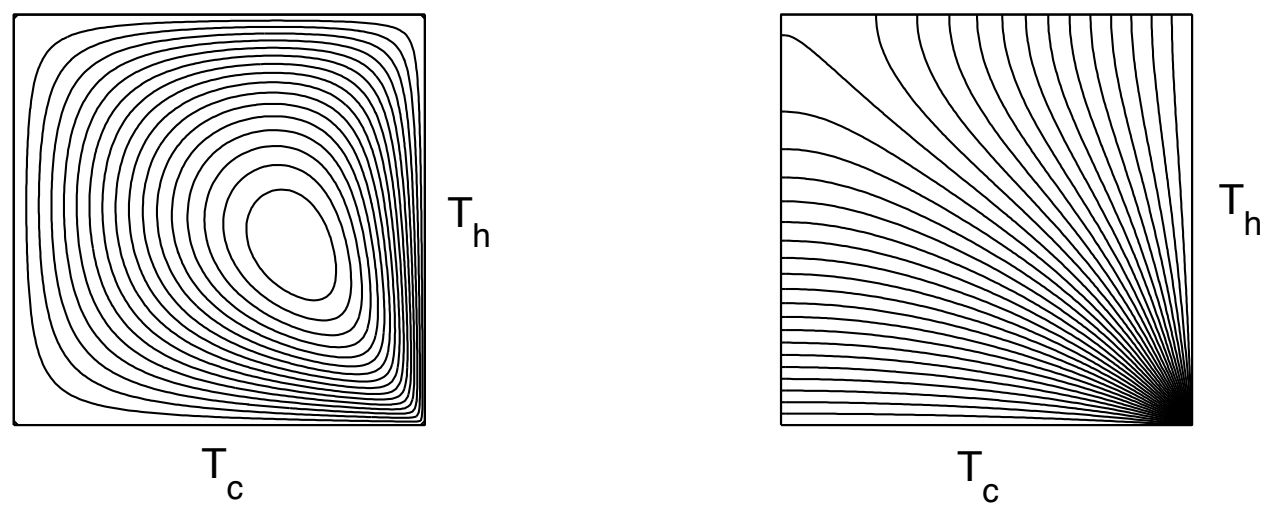

(f)
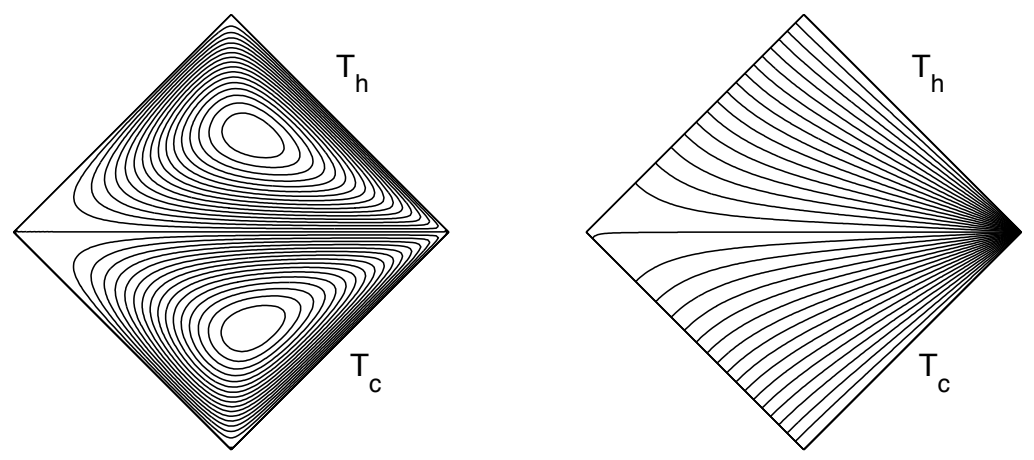

(g)
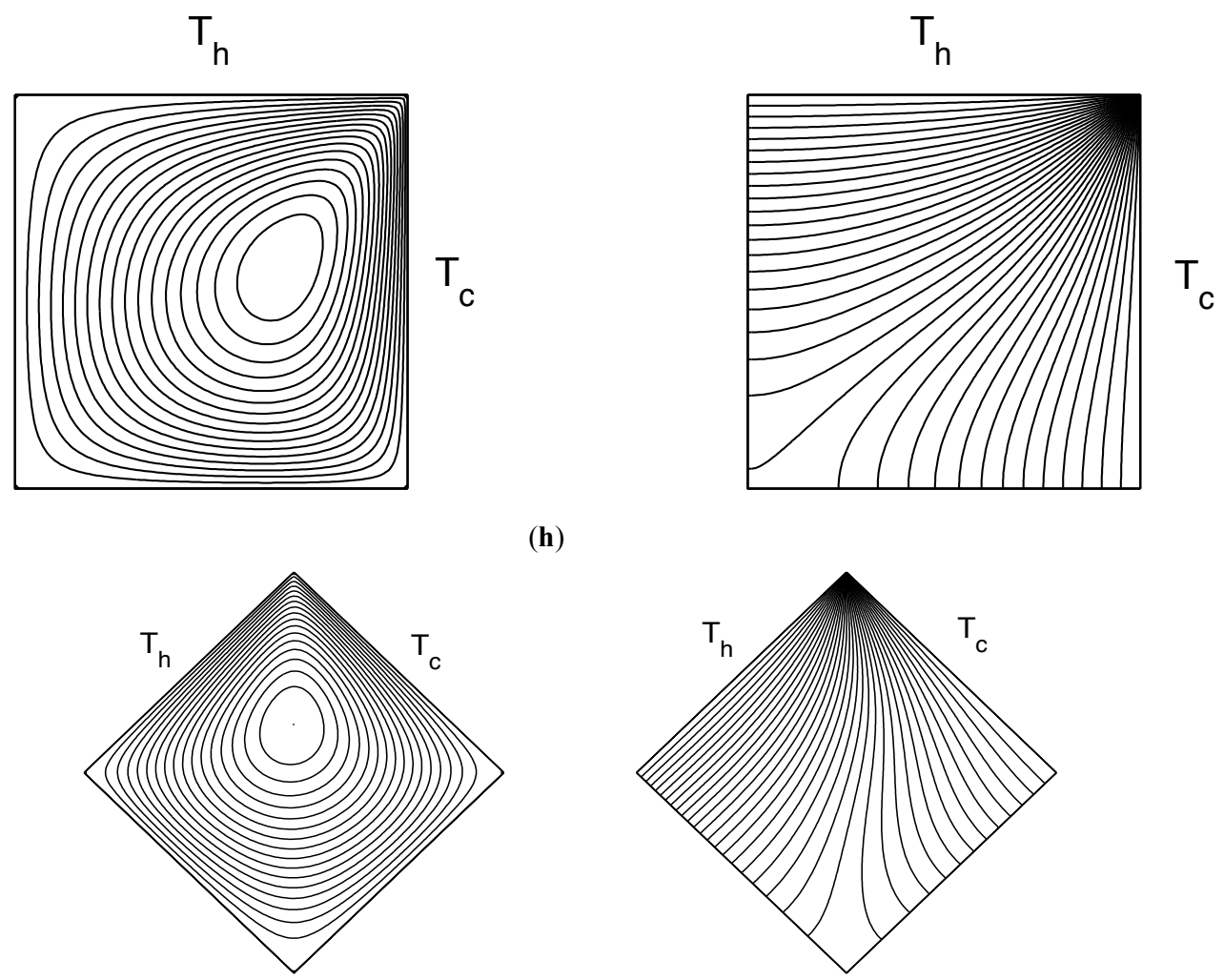

(h)

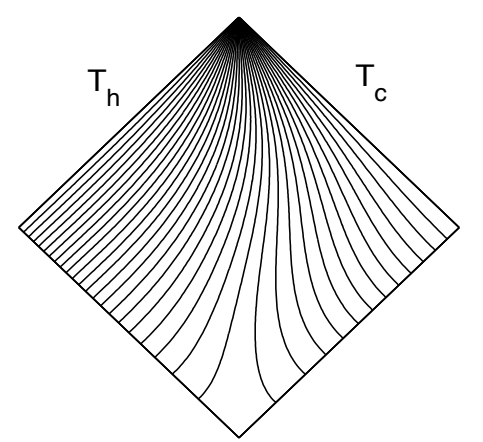

Fig. (2). Streamlines and isotherms for $R a=10$ and (a) $\varphi=0^{\circ} ;(\mathbf{b}) \quad \varphi=45^{\circ} ;$ (c) $\varphi=90^{\circ} ;(\mathbf{d}) \quad \varphi=135^{\circ} ;(\mathbf{e}) \quad \varphi=180^{\circ} ;(\mathbf{f}) \quad \varphi=225^{\circ}$; (g) $\varphi=270^{\circ}$; and (h) $\varphi=315^{\circ}$. 
(a)
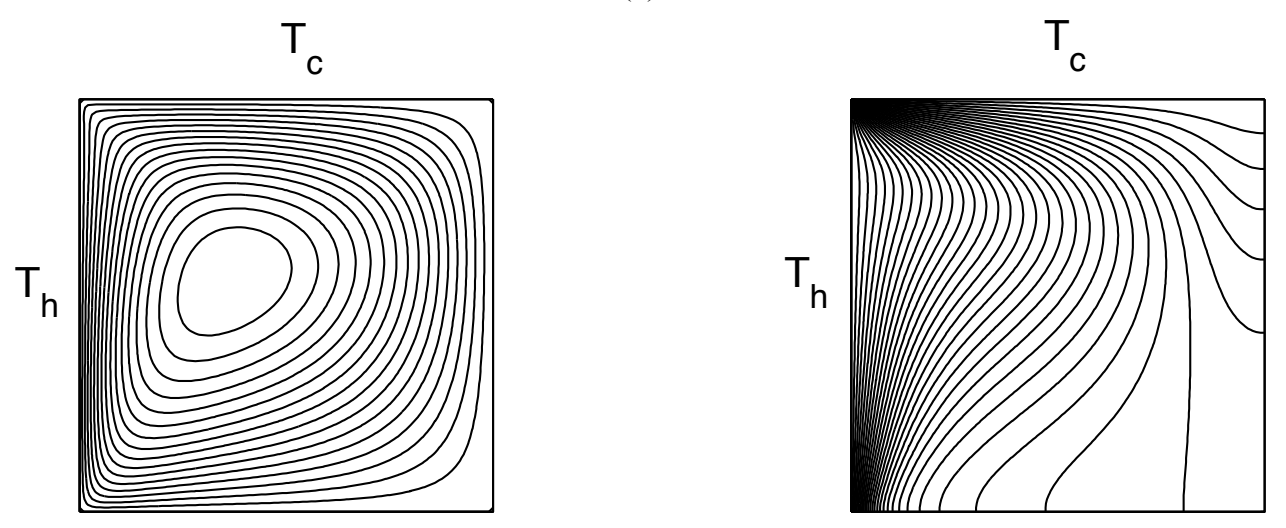

(b)
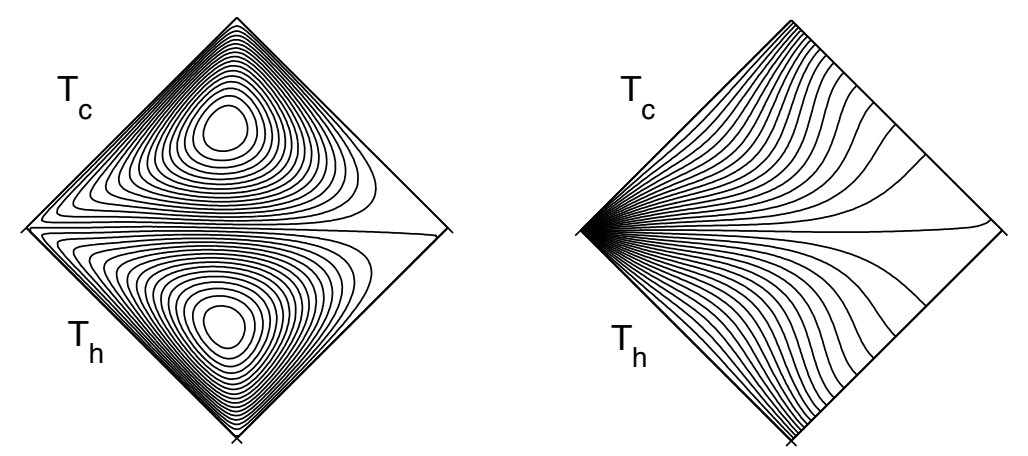

(c)
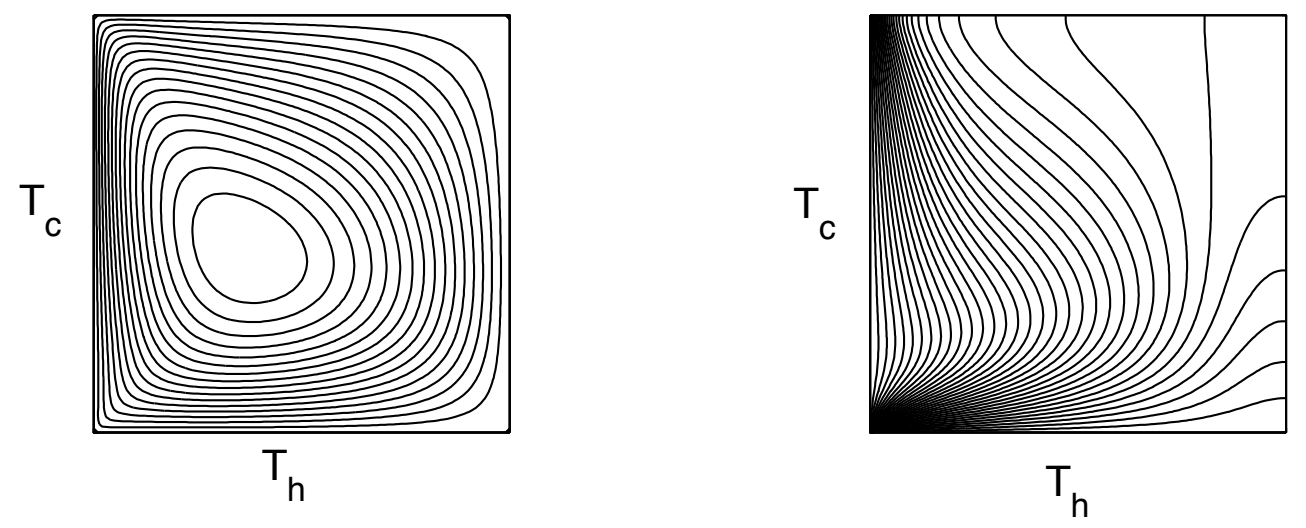

(d)
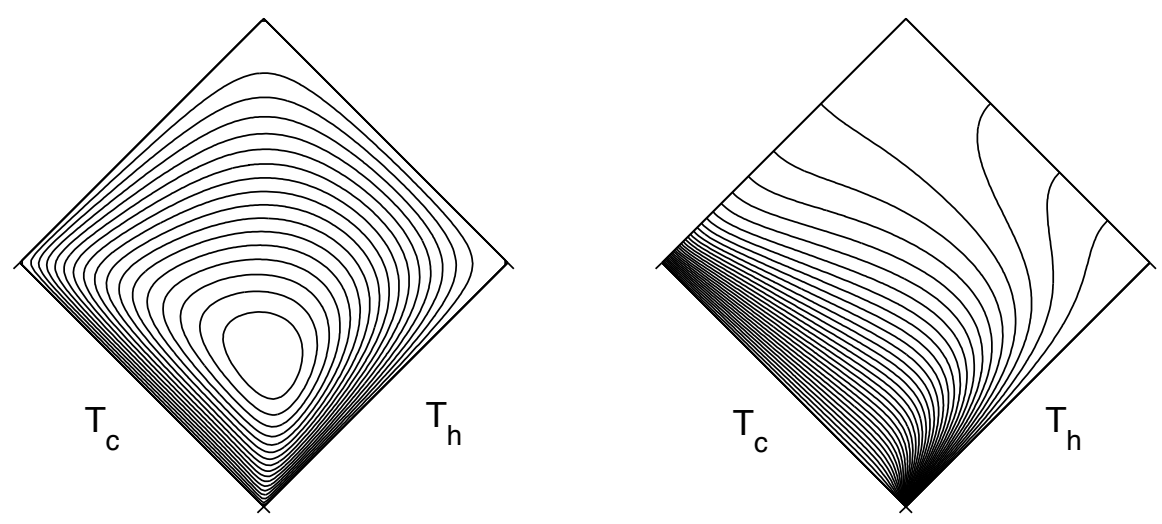
(e)

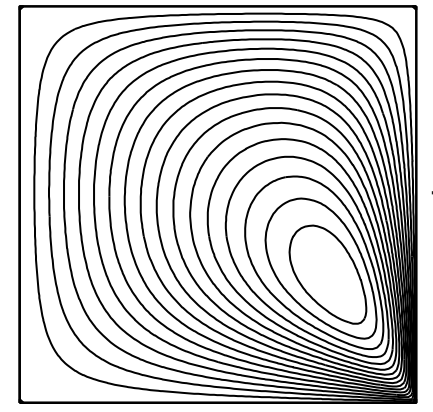

$\mathrm{T}_{\mathrm{c}}$

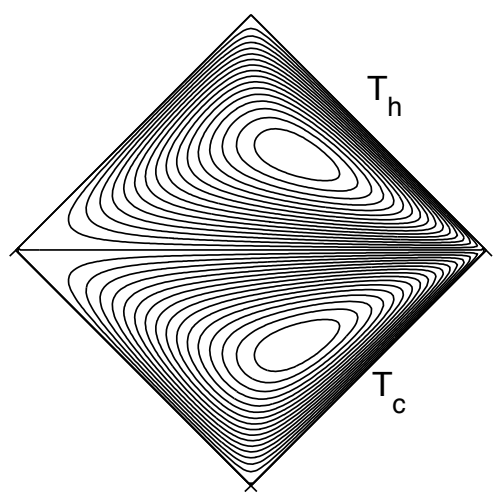

$(\mathbf{g})$
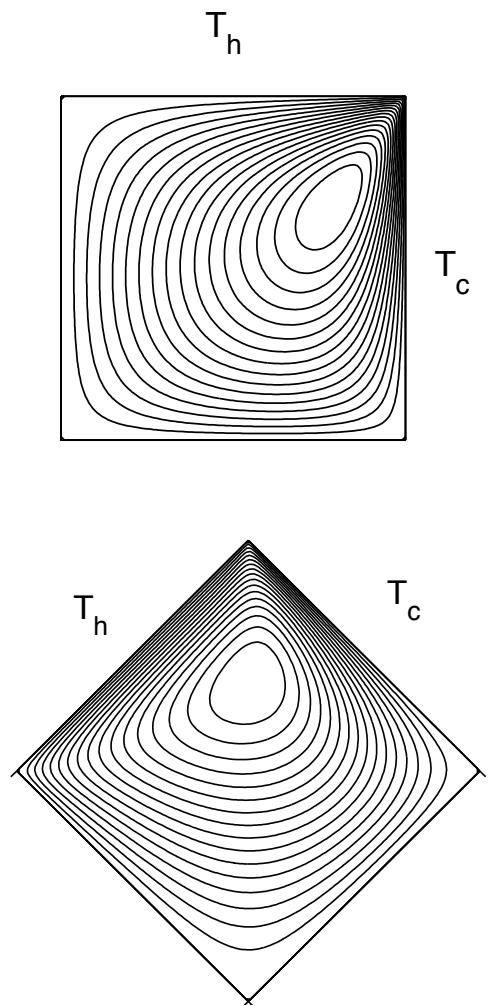

(f)

(h)

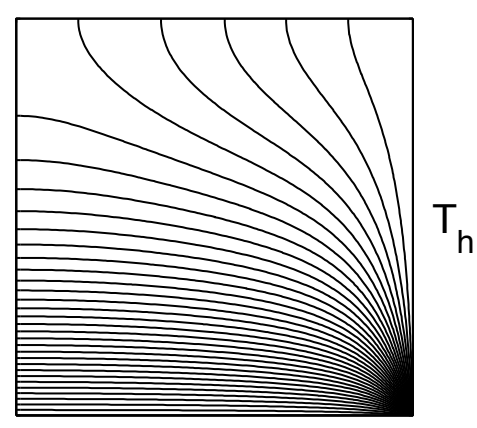

$\mathrm{T}_{\mathrm{C}}$
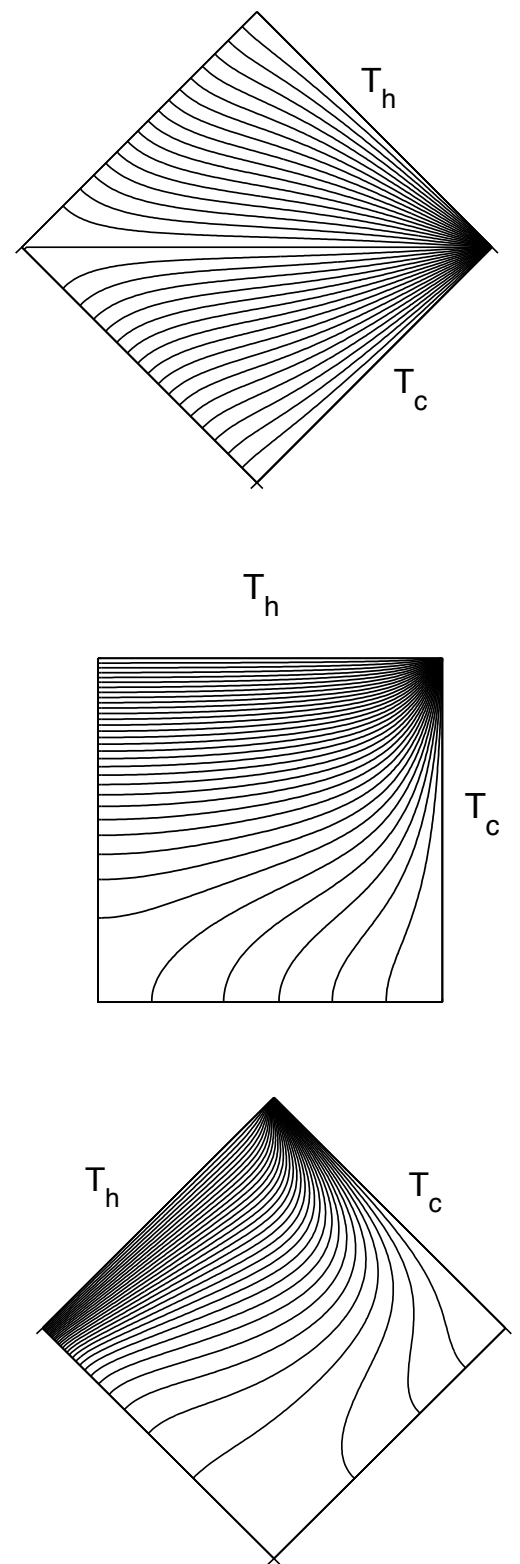

Fig. (3). Streamlines and isotherms for $R a=100$ and (a) $\varphi=0^{\circ} ;$ (b) $\varphi=45^{\circ} ;$ (c) $\varphi=90^{\circ} ;(\mathbf{d}) \quad \varphi=135^{\circ} ;(\mathbf{e}) \quad \varphi=180^{\circ} ;(\mathbf{f})$ $\varphi=225^{\circ} ;(\mathbf{g}) \quad \varphi=270^{\circ}$; and (h) $\varphi=315^{\circ}$. 


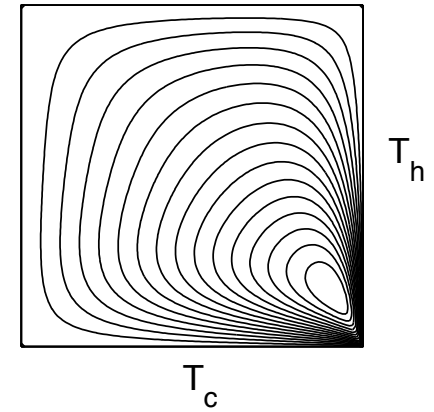

Fig. (4). Streamlines and isotherms for $R a=1000, \varphi=180^{\circ}$.

(a)

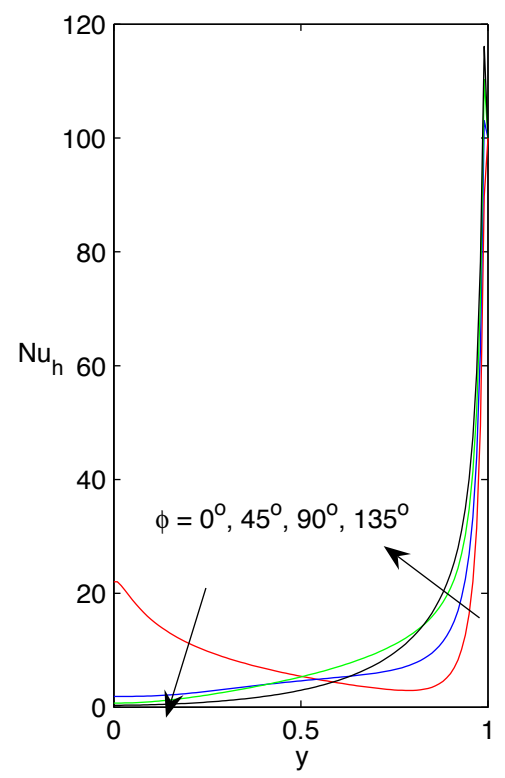

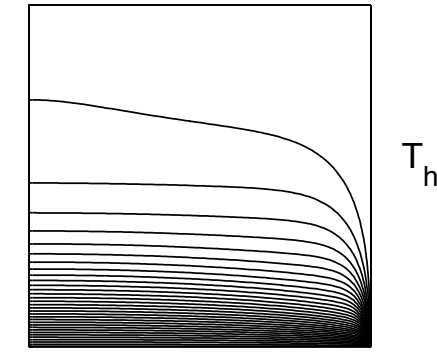

$T_{c}$

\section{h}

(b)

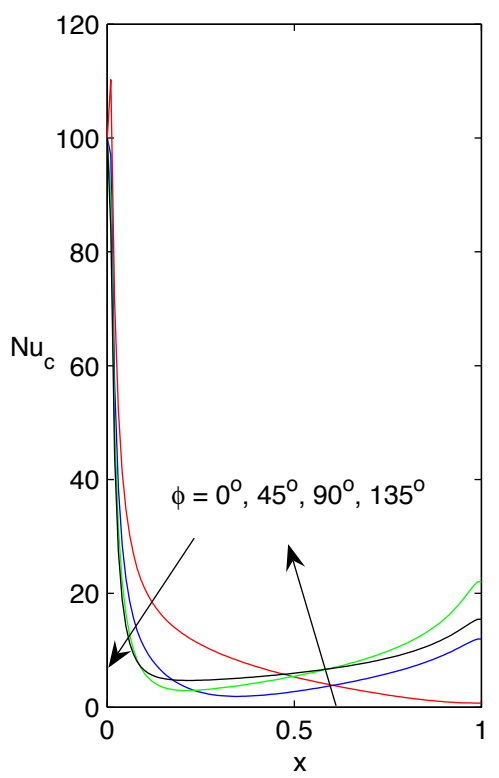

Fig. (5). Variation of ( a) $N u_{h}$ and (b) $N u_{c}$ for $R a=100$ and different values of $\phi$.

(a)

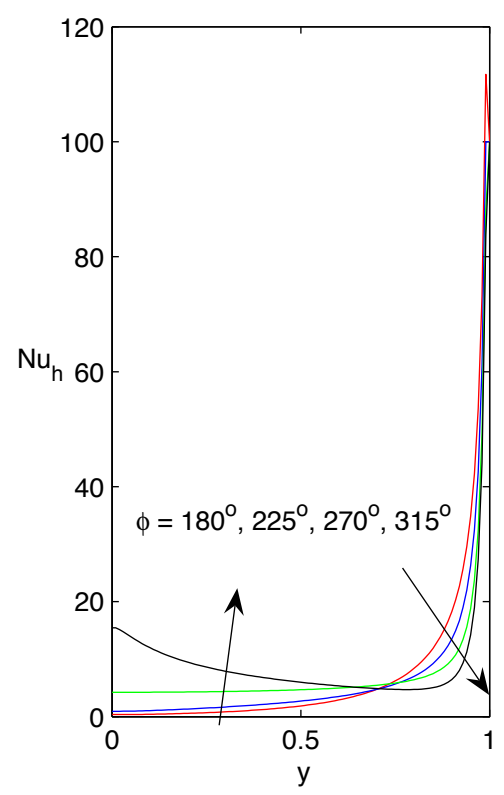

(b)

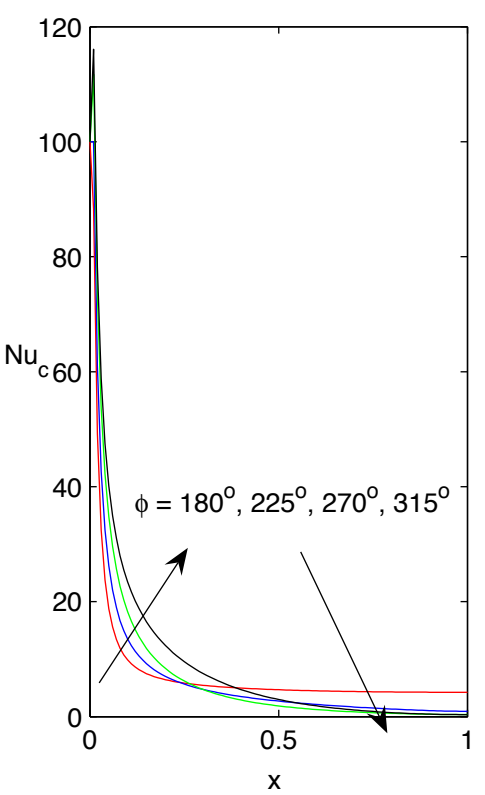

Fig. (6). Variation of (a) $N u_{h}$ and (b) $N u_{c}$ for $R a=100$ and different values of $\phi$. 
Table 2. Average of Nusselt Number $\overline{N u}_{h}$ for the Heated Wall Using $R a=100$

\begin{tabular}{|c|c|c|c|c|c|c|c|c|}
\hline$\varphi$ & $0^{\circ}$ & $45^{\circ}$ & $90^{\circ}$ & $135^{\circ}$ & $180^{\circ}$ & $225^{\circ}$ & $270^{\circ}$ & $315^{\circ}$ \\
\hline $26 \times 26$ & 8.2636 & 5.3705 & 8.2240 & 7.4053 & 5.6450 & 5.1780 & 6.0722 & 7.7828 \\
\hline $51 \times 51$ & 9.2162 & 6.2760 & 9.2008 & 8.4700 & 6.6573 & 6.0649 & 6.8935 & 8.6699 \\
\hline $101 \times 101$ & 10.1319 & 8.1912 & 10.1247 & 9.4311 & 7.6072 & 6.9500 & 7.7297 & 9.5335 \\
\hline Richardson extrapolation & 10.4371 & 8.8296 & 10.4326 & 9.7514 & 7.9238 & 7.2450 & 8.0084 & 9.8213 \\
\hline
\end{tabular}

Table 3. Average of Nusselt Number $\overline{N u}_{c}$ for the Cold Wall Using $R a=100$

\begin{tabular}{|c|c|c|c|c|c|c|c|c|}
\hline$\varphi$ & $0^{\circ}$ & $45^{\circ}$ & $90^{\circ}$ & $135^{\circ}$ & $180^{\circ}$ & $225^{\circ}$ & $270^{\circ}$ & $315^{\circ}$ \\
\hline $26 \times 26$ & 8.2240 & 5.3705 & 8.2636 & 7.7828 & 6.0722 & 5.1780 & 5.6450 & 7.4053 \\
\hline $51 \times 51$ & 9.2008 & 6.2759 & 9.2163 & 8.6698 & 6.8935 & 6.0650 & 6.6573 & 8.4699 \\
\hline $101 \times 101$ & 10.1247 & 8.1576 & 10.1319 & 9.5335 & 7.7297 & 6.9503 & 7.6072 & 9.4311 \\
\hline Richardson extrapolation & 10.4326 & 8.7848 & 10.4371 & 9.8214 & 8.0084 & 7.2454 & 7.9238 & 9.7515 \\
\hline
\end{tabular}

\section{CONCLUSIONS}

In this paper, we have numerically analyzed the steady natural convection flow in an inclined square cavity filled with a porous medium and isothermally heated from one side wall and isothermally cooled from an adjacent wall. The influence of the angle of inclination on the flow field and the energy transport is investigated for a range of angles of inclination, namely, $0^{\circ} \leq \phi \leq 315^{\circ}$ and for $R a=10,100$ and 1000. Because of the presence of the adjacent isothermal walls, this geometry represents different natural convection problems and exhibits some interesting fluid flow and energy transport mechanisms. It should be noted that the flow field becomes unstable for $R a=1000$ when the inclination angles are different from $\phi=180^{\circ}$. The minimum value of the average Nusselt numbers are attained at $\phi=225^{\circ}$ when $R a=100$, and in this case, conduction is dominant and the effect of $R a$ becomes insignificant, as can be observed from Tables 2 and 3. To this end, it should be notedthat we have adopted the Darcy model here but the paper can be extended to the Brinkman-extended non-Darcy model.

\section{NOMENCLATURE}

$g=$ Magnitude of the acceleration due to gravity, $\mathrm{m} / \mathrm{s}^{2}$

$h=$ Heat transfer coefficient, $\mathrm{W} / \mathrm{m}^{2} \mathrm{k}$

$k=$ Thermal conductivity, $\mathrm{W} / \mathrm{mK}$

$K=$ Permeability, $\mathrm{m}^{2}$

$L=$ Width of the square cavity, $\mathrm{m}$

$N u_{h}=$ Local Nusselt number from the heated wall

$\bar{N} u_{h}=$ Average Nusselt number from the heated wall
$N u_{c}=$ Local Nusselt number from the colded wall

$\bar{N} u_{c}=$ Average Nusselt number from the colded wall

$R a=$ Rayleigh number for a porous medium

$T=$ Temperature, $\mathrm{K}$

$T_{h} \quad=\quad$ Temperature for hot wall, $\mathrm{K}$

$T_{c}=$ Temperature for cold wall, $\mathrm{K}$

$T_{0} \quad=$ Volume average of the temperature over the fluid, $\mathrm{K}$

$u=$ Filtration velocity along $x-$ axis, $\mathrm{m} / \mathrm{s}$

$U=$ Dimensionless velocity along $X$-axis

$v=$ Filtration velocity along $y-$ axis, $\mathrm{m} / \mathrm{s}$

$V=$ Dimensionless velocity along $Y-$ axis

$x=$ Coordinate measured along the lower horizontal wall, $\mathrm{m}$

$X=$ Dimensionless coordinate in the horizontal direction

$y=$ Coordinate measured along the hot vertical wall, $\mathrm{m}$

$Y=$ Dimensionless coordinate in the vertical direction

\section{Greek Symbols}

$\beta=$ Dimensionless modified thermal capacity ratio

$\alpha_{m}=$ Effective thermal diffusivity of the porous medium, $m^{2} / s$

$\phi \quad=$ Inclination angle, degree 


$$
\begin{array}{ll}
v & =\text { Dynamic viscosity, } \mathrm{m}^{2} / \mathrm{s} \\
\theta & =\text { Dimensionless temperature } \\
\psi & =\text { Dimensionless stream function } \\
\rho & =\text { Density of the fluid, } \mathrm{kg} / \mathrm{m}^{3} \\
\varepsilon & =\text { Convergence criterion }
\end{array}
$$

\section{Subscrips}

$$
\begin{array}{ll}
c & =\text { Cold wall } \\
h & =\text { Hot wall }
\end{array}
$$

\section{ACKNOWLEDGEMENTS}

The authors wish to express their sincere thanks to the reviewers for their valuable comments and suggestions. In additions, C. Revnic and T. Grosan wish to express their thanks to UEFISCSU Grant PN-II-ID 525/2007.

\section{REFERENCES}

[1] A. Nakayama, PC-Aided Numerical Heat Transfer and Convective Flow. Tokyo : CRC Press 1995.

[2] D.A. Nield, and A. Bejan, Convection in Porous Media. $3^{\text {rd }}$ ed. New York: Springer, 2006.

[3] D.B. Ingham, and I. Pop, Eds. Transport Phenomena in Porous Media. Oxford: Elsevier, 2005.

[4] K. Vafai, Ed. Hanbook of Porous Media. $2^{\text {nd }}$ ed. Boca Raton: Taylor\& Francis, 2005.

[5] M.J.S. de Lemos, Turbulence in Porous Media: Modeling and Applications. Oxford: Elsevier, 2006.

[6] P. Vadasz, Ed. Emerging Topics in Heat and Mass Transfer in Porous Media. New York: Springer, 2008.

[7] A. Bejan, "On the boundary layer regime in a vertical enclosure filled with a porous medium", Lett. Heat Mass Transf., vol. 6, pp. 93-102, 1979.

[8] B. Goyeau, J.P. Songbe, and D. Gobin, "Numerical study of double-diffusive natural convection in a porous cavity using the Darcy-Brinkman formulation", Int. J. Heat Mass Transf., vol. 39, pp. 1363-1378, 1996.

[9] R.J. Gross, M.R. Bear, and C.E. Hickox, The application of fluxcorrected transport (FCT) to high Rayleigh number natural convection in a porous medium. In: Proceedings of $8^{\text {th }}$
International on Heat Transfer Conference, San Francisco, CA, 1986.

[10] D.M. Manole, and J.L. Lage, "Numerical benchmark results for natural convection in a porous medium Cavity", In: Heat and Mass Transfer in Porous Media, ASME Conf., HTD-vol. 216, 1992, pp. 55-60.

[11] N.H. Saeid, and I. Pop, "Natural convection from a discrete heater in a square cavity filled with a porous medium", J. Porous Media, vol. 8, pp. 55-63, 2005.

[12] A.C. Baytas, and I. Pop, "Free convection in a square porous cavity using a thermal nonequilibrium model", Int. J. Thermal Sci., vol. 41, pp. 861-870, 2002.

[13] R. Anderson, and G. Lauriant, "The horisontal natural convection boundary layer regim in a closed Cavity", In: Proceedings of the Eighth International Heat Transfer Conference 1. San Francisco, CA, 1996, pp. 1453-1458.

[14] S. Kımura, and A. Bejan, "Natural convection in a differentially heated corner region", Phys. Fluids, vol. 28, pp. 2980-2989, 1995.

[15] M.M. Ganzarolli, and L.F. Milanez, "Natural convection in rectangular enclosure heated from below and symmetrically cooled from the sides", Int. J. Heat Mass Transf., vol. 38, pp. 1063-1073, 1995.

[16] O. Aydin, A. Unal, and T. Ayhan, "A numerical study on buoyancy-driven flow in an inclined square enclosure heated and cooled on adjacent walls", Numer. Heat Transf., vol. 36, pp. 585599, 1999a.

[17] O. Aydin, A. Unal, and T. Ayhan, "Natural convection in rectangular enclosure heated from one side and cooled from the ceiling", Int. J. Heat Mass Transf., vol. 42, pp. 2345-2355, 1999b.

[18] M. Rahman, and M.A.R. Sharif, "Numerical study of laminar natural convection in inclined rectangular enclosures of various aspect ratios", Numer. Heat Transf. Part A, vol. 44, pp. 355-373, 2003.

[19] M.C. Ece and, E. Büyük, "Natural convection flow under a magnetic field in an inclined rectangular enclosure heated and cooled on adjacent walls", Fluid Dyn. Res., vol. 38, pp. 564-590, 2006.

[20] G.D. Smith, Numerical Solution of Partial Differential Equations. Finite Difference Method, Oxford University Press, Oxford, 2004.

[21] T. Grosan, C. Revnic, I. Pop, and D.B. Ingham, "Magnetic field and internal heat generation effects on the free convection in a rectangular cavity filled with a porous medium", Int. J. Heat Mass Transfer, vol. 52, pp. 1525-1533, 2009.

[22] C. Revnic, T. Grosan, I. Pop, and D.B. Ingham, "Free convection in a square cavity filled with a bidisperse porous medium", Int. J. Thermal Sci. [Available online 14 March 2009].

[23] A.C. Baytas, and I. Pop, "Free convection in oblique enclosures filled with a porous medium", Int. J. Heat Mass Transf., vol. 42, pp. 1047-1057,1999 